\title{
A retrospective study of 34 patients with unicentric and multicentric Castleman's disease: Experience from a single institution
}

\author{
JIN-PENG JIANG ${ }^{1,2^{*}}$, XIAO-FEI SHEN ${ }^{2 *}$, JUN-FENG DU $^{3}$ and WEN-XIAN GUAN ${ }^{2}$ \\ ${ }^{1}$ Department of Rehabilitation Medicine, General Hospital of Beijing Military Command, Beijing 100700; ${ }^{2}$ Department of \\ General Surgery, Affiliated Drum Tower Hospital of Nanjing University Medical School, Nanjing, Jiangsu 210008; \\ ${ }^{3}$ Department of General Surgery, General Hospital of Beijing Military Command, Beijing 100700, P.R. China
}

Received October 27, 2015; Accepted April 13, 2017

DOI: $10.3892 / \mathrm{ol} .2017 .7625$

\begin{abstract}
The aim of the present study was to share the experience of a single institute in the diagnosis, use of accessory examinations and treatment strategies of Castleman's disease (CD). The present study analyzed 34 patients (13 males and 21 females) with CD who were hospitalized between January 2006 and September 2014. The patients were divided into two groups based on the anatomical distribution of the disease: Unicentric CD (UCD) and multicentric CD (MCD). Histological data was obtained from lymph node biopsies. All clinical data were acquired by reviewing patients' medical records and contacting patients by telephone. A total of 27 patients had UCD and 7 patients had MCD. All 27 patients with UCD with benign symptoms underwent complete diagnostic surgical resection and survived, with the exception of 1 patient who succumbed to pancreatic head carcinoma 13 months after surgery. A total of 7 patients with MCD presented with systemic symptoms and 2 of these patients declined treatment following the definite diagnosis of CD. The remaining 5 patients were treated with various strategies, including surgical resection and further glucocorticoid treatment, intravenous siltuximab, rituximab in combination with cyclophosphamide, doxorubicin, vincristine and prednisone chemotherapy or hematopoietic stem cell transplantation. A total of 3 patients with MCD survived, with a median follow-up period of 69 months. The present study indicates that complete surgical resection is currently the standard treatment for UCD.
\end{abstract}

Correspondence to: Dr Wen-Xian Guan, Department of General Surgery, Affiliated Drum Tower Hospital of Nanjing University Medical School, 321 Zhongshan Road, Nanjing, Jiangsu 210008, P.R. China

E-mail: guan-wx@163.com

*Contributed equally

Key words: Castleman's disease, retroperitoneum, laparoscopic surgery, case series
Perioperative use of multidetector computed tomography and the laparoscopic approach have certain advantages in UCD. Molecular target therapy is effective in patients with stable MCD, and hematopoietic stem cell transplantation may be beneficial in certain patients with MCD and disease progression.

\section{Introduction}

Castleman's disease (CD) is a rare lymphoproliferative disorder characterized by enlarged hyperplastic lymph nodes. It has been six decades since the discovery of CD by Dr Benjamin Castleman, who described a patient with a solitary hyperplastic mediastinal lymph node in 1954 (1). With the development of clinical examination and imaging technology, numerous previous studies have investigated $\mathrm{CD}$, including the pathophysiology, associated inflammatory mediators and molecular therapies $(2,3)$. Generally, CD can be categorized into two subtypes based on the anatomical distribution of disease: Unicentric CD (UCD) and multicentric CD (MCD). UCD is the most common form, with localized disease and usually being the hyaline vascular histological type (4), whereas MCD is a much more aggressive form with a plasma cell variant pattern histologically (3). Pathological biopsy is required for the diagnosis of $\mathrm{CD}$ when a patient presents with lymphadenopathy, as UCD is often asymptomatic and MCD is frequently accompanied by systemic manifestations, including fever, fatigue, edema and weight loss (5). Laboratory abnormalities have been observed in patients with $\mathrm{CD}$, including anemia, leukocytosis, thrombocytosis, hypergammaglobulinemia, hypoalbuminemia and increased C-reactive protein expression levels (5); however, these are usually assigned to a differential diagnosis that includes rheumatic diseases and lymphoma. Notably, the detection of dysregulated plasma interleukin (IL)-6 level may be useful upon laboratory examination, as previous studies have shed light on the critical role of IL-6 in the disease process $(2,6,7)$.

The aim of the present study was to investigate the diagnosis, use of accessory examinations and treatment strategies in 34 patients with CD treated in Drum Tower Hospital of Nanjing University Medical School (Nanjing, China). The 
present study also identified recent advances in elucidating the pathophysiology of $\mathrm{CD}$, and discussed available treatment options to improve the understanding of this uncommon disease.

\section{Patients and methods}

Patients. A total of 34 patients with $\mathrm{CD}$ who were treated at Drum Tower Hospital of Nanjing University Medical School (Nanjing, China) between January 2006 and September 2014 were identified from the pathological database. A total of 13 patients were men and 21 were women, with a median age of 47 years and a range of 24-71 years. Histological data were obtained from lymph node biopsies to confirm the diagnosis of CD. All clinical data were acquired by reviewing medical records and contacting patients by telephone. Although the association between human immunodeficiency virus (HIV) infection and CD has been observed in a previous study (8), the serological test results for HIV in all the patients in the present study were negative. Written informed consent was obtained from all study participants or their legal guardian prior to enrollment in the present study. All procedures performed in the present study involving human participants were in accordance with the ethical standards of the Human Subjects Institutional Committee of Drum Tower Hospital, and with the 1964 Helsinki Declaration and its later amendments.

Category definitions. Based on the anatomical distribution of the disease, patients were divided into two groups: The more common UCD and the relatively less common MCD. As aforementioned, the UCD group consisted of patients who displayed histological evidence of $\mathrm{CD}$ in 1 single group of lymph nodes without clinical or radiological evidence of adenopathy elsewhere. Patients with MCD displayed histological evidence of $\mathrm{CD}$ in $\geq 1$ group of lymph nodes and radiological or clinical evidence of additional adenopathy. According to the histological criteria proposed by Keller et al (4), CD was further classified into two types: The hyaline vascular (HV) type and the plasma cell (PC) type. Lymph nodes with characteristics intermediate between $\mathrm{HV}$ and PC were categorized as a mixed type.

Data collection. Relevant clinical data, including gender, chief complaint, clinical presentation, and lymph node distribution; laboratory data including erythrocyte sedimentation rate (ESR), C-reactive protein (CRP) level, leukocyte number, and albumin/globulin ratio; radiological and pathological data were collected to evaluate disease progression and treatment response. Different treatment options, including surgery, chemotherapy and radiation therapy, were recorded and assessed for clinical efficacy in terms of post-treatment prognoses.

\section{Results}

Location and histological features. There was a total of 27 patients with UCD and 7 with MCD. The median ages of the patients with UCD and MCD were 48 and 46 years, respectively. By definition, UCD was localized to one site. Of the 27 unicentric patients, 9 presented with UCD of the retroperitoneum (33.3\%), 6 with UCD of the neck (22.2\%), 4 with UCD of the mediastinum (14.8\%), 3 with UCD of the groin (11.1\%), 2 with UCD of the pelvic cavity (7.4\%), 2 with $\mathrm{UCD}$ of the armpit (7.4\%) and 1 with UCD of the mesentery (3.7\%). Among the patients with MCD, 6 patients presented with MCD of the armpit. HVCD was identified in 22 patients $(88 \%)$ with UCD and in 3 patients with MCD (12\%). Conversely, PCCD was observed in 5 patients $(55.5 \%)$ with UCD and in 4 patients with MCD (44.5\%). No mixed variants were identified in these patients.

Clinical manifestations and signs. The relevant major symptoms in the 34 patients with CD are presented in Table I. In general, 14 patients with UCD were detected on routine examination without displaying symptoms and the anatomical regions involved included the retroperitoneum, mediastinum, mesentery and pelvic cavity. A total of 11 patients with UCD had swollen lymph nodes and were admitted to hospital for lymph node biopsy, and the other 2 patients with UCD were identified by lymph node biopsy during radical surgery for liver cancer and pancreatic cancer, which were initially recognized as lymph node metastasis. Of the 7 patients with MCD, 5 patients displayed systemic manifestations, including fever and fatigue, and 1 patient had breathing difficulties induced by enlarged lymph nodes compressing the trachea. The remaining 2 patients did not present with any clinical symptoms, and were initially identified by routine examination of computed tomography (CT) scans.

Laboratory and radiological examinations. Numerous frequently recorded abnormal laboratory values were analyzed in patients with UCD and MCD. In patients with UCD, an elevated immunoglobulin $\mathrm{G}$ level was observed in 6 patients $(22.2 \%)$, reduced hemoglobin levels in 3 patients $(11.1 \%)$, a reduced leukocyte number in 2 patients (7.4\%), and an elevated C-reactive protein expression level and erythrocyte sedimentation rate in $5(18.5 \%)$ and 6 patients $(22.2 \%)$, respectively. An elevated immunoglobulin $\mathrm{G}$ expression level was revealed in 5 patients (71.4\%) with MCD. An elevated erythrocyte sedimentation rate and C-reactive protein expression level were demonstrated in 5 patients. Compared with patients with $\mathrm{UCD}$, skin lesions and numbness of the extremities were also observed in 2 and 1 patient, respectively, in the MCD group. These 2 patients with skin lesions were also diagnosed with polyneuropathy, organomegaly, endocrinopathy, monoclonal gammopathy and skin changes syndrome.

All patients with UCD underwent CT scans (Fig. 1), which displayed an atypical homogeneous soft tissue mass with moderate enhancement following injection of contrast material. A total of 12 patients with UCD also underwent multidetector computed tomography (MDCT) angiography (9) to clarify the association between enlarged tissue, blood vessels and peripheral vital organs (Fig. 2). MCD was characterized by predominant lymphadenopathy, which involved peripheral lymph nodes. Furthermore, patients in the MCD group presented with effusion on CT scans, involving the chest (3 patients), the peritoneum (1 patient) and the pericardium (2 patients). A single patient underwent position emission tomography (PET)-CT examination, and multiple intrahepatic low densities were revealed with markedly increased 
Table I. Clinical manifestations and indicators.

\begin{tabular}{|c|c|c|}
\hline Category & UCD & MCD \\
\hline \multicolumn{3}{|l|}{ Histological feature, $n$} \\
\hline HV type & 22 & 3 \\
\hline PC type & 5 & 4 \\
\hline Mixed-type & 0 & 0 \\
\hline \multicolumn{3}{|l|}{ Gender } \\
\hline Male/female, $\mathrm{n}$ & $9 / 18$ & $4 / 3$ \\
\hline Median age, years & 48 & 46 \\
\hline \multicolumn{3}{|l|}{ Chief complaint, $\mathrm{n}$} \\
\hline Routine examination & 14 & 2 \\
\hline Accidental touch & 11 & 1 \\
\hline Compression symptoms & 0 & 1 \\
\hline Other signs & 2 & 0 \\
\hline \multicolumn{3}{|l|}{ Clinical presentation, $\mathrm{n}$} \\
\hline Fever & 0 & 5 \\
\hline Fatigue & 0 & 6 \\
\hline Weight loss & 0 & 3 \\
\hline Edema & 0 & 1 \\
\hline Anemia & 3 & 5 \\
\hline ESR elevated & 3 & 5 \\
\hline CRP elevated & 5 & 5 \\
\hline Albumin/globulin decreased & 6 & 5 \\
\hline Decreased leukocytes & 2 & 4 \\
\hline Pleural effusion & 0 & 3 \\
\hline Ascites & 0 & 1 \\
\hline Pericardial effusion & 0 & 2 \\
\hline Skin lesion & 0 & 2 \\
\hline Numbness of extremities & 0 & 1 \\
\hline Hepatosplenomegaly & 0 & 3 \\
\hline \multicolumn{3}{|l|}{ Lymph node distribution, $\mathrm{n}$} \\
\hline Neck & 6 & 3 \\
\hline Mediastinum & 4 & 3 \\
\hline Retroperitoneum & 9 & 4 \\
\hline Armpit & 2 & 6 \\
\hline Pelvic cavity & 2 & 1 \\
\hline Groin & 3 & 5 \\
\hline Mesentery & 1 & 0 \\
\hline
\end{tabular}

ESR, erythrocyte sedimentation rate; CRP, C-reactive protein; UCD, unicentric Castleman's disease; MCD, multicentric Castleman's disease; HV, hyaline vascular; PC, plasma cell.

metabolism. The largest standardized uptake value (SUV) was 11.3 and the median SUV was 8.5. The present study also observed enlarged lymph nodes in the mediastinal, bilateral axillary, hilar and groin regions, with a median SUV of 3.1.

Treatment and prognosis. A total of 22 patients with UCD underwent laparotomy for complete resection of the tissue mass, and 5 patients underwent surgery using the laparoscopic approach (the retroperitoneum in 4 and the mesentery in 1). None of the patients with UCD received further chemotherapy
A

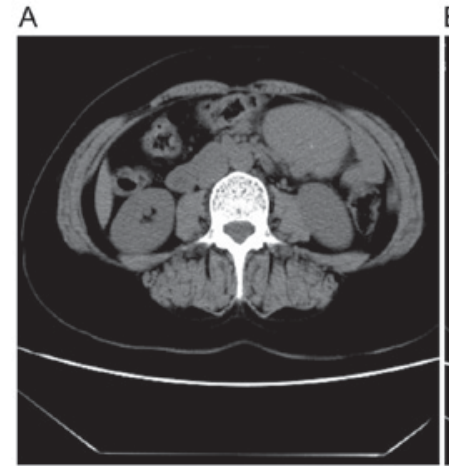

B

Figure 1. Computed tomography scan of the abdomen demonstrating the location of the enlarged tissue mass in a representative patient. The computed tomography scan results of a representative patient with Castleman's disease were shown. An atypical homogeneous soft tissue mass (A) was presented in the middle of the left abdomen. This moderate soft tissue mass also showed moderate enhancement following injection of contrast material (B), which was in close relation to spleen and kidney.
A

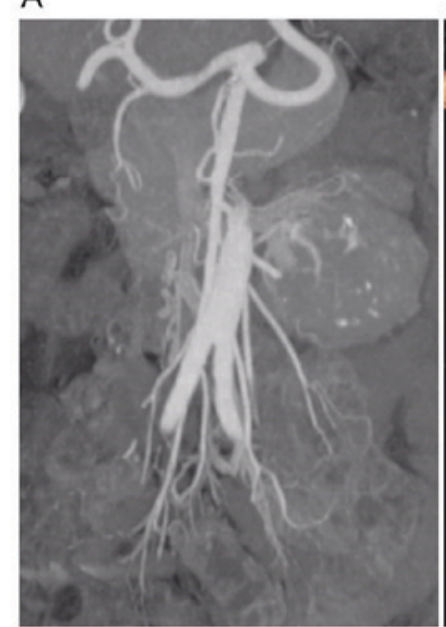

B

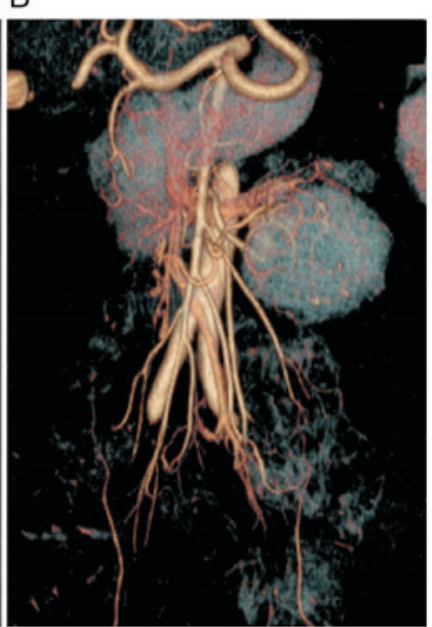

Figure 2. Multidetector computed tomography demonstrating blood vessels of an enlarged tissue mass in a representative patient. Multidector computed tomography angiography results of a representative patient with Castleman's disease were shown. The association between enlarged tissue, blood vessels and peripheral vital organs such as the pancreas was presented (A), and three-dimensional reconstruction (B) further showed the clear relationship between abdominal aorta and blood vessels related to the enlarged tissue.

or radiotherapy, as there was no evidence of CD during the follow-up period. All patients survived with the exception of 1 patient who succumbed to pancreatic cancer. The mean follow-up period was $49.2 \pm 31.5$ months (mean \pm standard error of the mean).

In the 7 patients with MCD, 2 underwent cervical lymph node dissection and retroperitoneal lymph node dissection, respectively, and received glucocorticoid treatment. Patient 1 underwent cervical lymph node dissection and survived with a follow-up period of 12 months. Patient 2 received retroperitoneal lymph node dissection and succumbed 2 months after surgery. A total of 5 patients underwent lymph node biopsy and 2 declined treatment following the definite diagnosis of CD. No follow-up data was available for these 2 patients. The remaining 3 patients received various treatments: 1 patient received intravenous siltuximab at a dose of $12 \mathrm{mg} / \mathrm{kg}$ every 
3 weeks for 42 weeks and survived, and 1 patient received weekly intravenous rituximab $\left(375 \mathrm{mg} / \mathrm{m}^{2} /\right.$ dose $)$ combined with cyclophosphamide, doxorubicin, vincristine and prednisolone chemotherapy (CHOP for 8 cycles). The remaining patient was administered methylprednisolone ( $1 \mathrm{~g} /$ day x 5 days) combined with Imuran $(2 \mathrm{mg} / \mathrm{kg}$ every day for 12 weeks) and hydroxyurea (40 mg/kg 2 times every week for 12 weeks) orally, and received autologous hematopoietic stem cell transplantation 3 months after failure to control the disease. These 3 patients survived with a median follow-up period of 69 months.

\section{Discussion}

$\mathrm{CD}$ is an uncommon disease that comprises a heterogeneous group of lymphoproliferative disorders (10). Currently, no guideline is available for this disease due to its low incidence and complicated pathophysiology. The diagnosis of CD is established by histological definition and further classified by centricity, with no specific relevant clinical features, and accessory examinations (11). The treatment of CD, particularly MCD, remains under investigation (2). The present study was a retrospective analysis of unicentric and multicentric CD involving 34 patients treated in Drum Tower Hospital of Nanjing University Medical School. Although numerous similar studies have also been performed with different numbers of patients, the present study attempted to develop a strategy using these previous experiences and aimed to provide useful data.

CD can occur at any age (12), and there are no ethnicity or gender differences with regard to the incidence of CD (12). Studies performed to examine age disparity demonstrated that the majority of patients with UCD were 30-50 years old, whereas patients with MCD were 50-70 years old (13). In the present study, the median age in the UCD group was 48 years, and 46 years in the MCD group. According to a previous study, all lymph nodes are susceptible to CD (13). The majority of lesions in UCD patients occur in the mediastinum, followed by other sites, including the neck, abdomen, retroperitoneum and axilla $(14,15)$. A similar pattern of distribution was observed in the present study, with the retroperitoneum being the most common site (33.3\%), followed by the neck (22.2\%) and mediastinum (14.8\%). A total of 6 cases of MCD involved the axilla and only 1 involved the cervical pelvis.

According to a previous study, the majority patients with UCD are asymptomatic and MCD is most commonly reported with systemic symptoms, which may be attributed to vIL-6 (a viral homologue of IL-6 encoded by Kaposi's sarcoma herpesvirus) (3), including fever, fatigue and weight loss. In the present study, half of the patients with UCD were identified by routine examination, and the other half were identified to have enlarged, painless lymph nodes. These patients did not demonstrate any symptoms, which was consistent with a previous report (3). A total of 2 patients who were admitted to hospital with other symptoms were diagnosed by lymph node biopsy during surgery for cancer. It was also revealed that nearly all patients with MCD had associated systemic symptoms, with fatigue being the most common (85.7\%), followed by fever (71.4\%). Therefore, when patients are admitted to hospital with a chronic inflammatory status, the diagnosis of CD should also be taken into consideration in addition to hematological tumors.
Abnormalities in the diagnostic examinations, including elevated C-reactive protein expression level and erythrocyte sedimentation rate, were mostly observed in the patients with MCD in the present study, which is consistent with the results of a previous study (3). The radiological characteristics of CD can also be observed in benign or malignant lymphomatous tumors and other mediastinal masses. Various histological subtypes demonstrated moderate differences on contrast-enhanced CT scans. HVCD (hyaline-vascular CD) and mixed-CD (mixed type of hyaline-vascular and plasma cell $\mathrm{CD}$ ) revealed marked enhancement in the arterial phase, whereas PCCD (plasma cell CD) demonstrated less enhancement in the arterial phase and delayed enhancement. These differences may be the result of increased angiogenesis in the HVCD and mixed-CD lesions. The use of PET-CT has improved the diagnosis of $\mathrm{CD}$, as it is able to identify all enlarged lymph nodes with low FDG uptake (16). A patient in the present study underwent PET-CT examination and multiple enlarged lymph nodes were observed. As this patient also presented with intrahepatic low density, which indicated hepatocellular carcinoma with lymph node metastasis, an inguinal lymph node biopsy was performed and the histological results revealed CD. Therefore, PET-CT may be useful for the evaluation of enlarged lymph nodes, but demonstrates less efficiency in the diagnosis of $\mathrm{CD}$. We also recommend MDCT as a regular accessory examination for patients with an enlarged tissue mass, as it has advantages in the perioperative evaluation of the association between blood vessels, tissue masses and the surrounding organs.

CD has three histological variants: HVCD, PCCD and a mixed type. HVCD has been reported in $90 \%$ of patients with $\mathrm{UCD}$, but rarely in patients with MCD, whereas PCCD has been reported in only $10 \%$ of patients with UCD and in $80-90 \%$ of patients with MCD (9). In the present study, the HV type was identified in $81.5 \%$ of patients with UCD, and the PC type was observed in $57.1 \%$ of patients with MCD which was slightly lower compared with one recent study from China (17). The histological characteristics of HVCD consisted of distinctive dysplasia follicles with regressed germinal centers and a broad mantle zone of lymphocytes, which formed a concentric ring (known as the 'onion-skin' arrangement) (18). Another important feature of HVCD is increased interfollicular vascularity with hyalinized vessels, which may penetrate the germinal center and result in inflammatory cell infiltration (19). The differential diagnosis of HVCD in histological samples includes thymoma and angioimmunoblastic differentiated lymphadenopathy (20). The histological features of PCCD are usually not so distinctive and differ from those of HVCD, with less interfollicular vascularity of hyalinized vessels and onion-skin arrangement. The majority of PCCD presents with proliferation of follicles and plasma cell infiltration accompanied by Russell bodies (21). The differential diagnosis of PCCD in histological samples includes autoimmune diseases, malignancies and reactive lymph node hyperplasia $(3,22)$. It should be noted that the histological type is of secondary importance compared with the unicentric or multicentric nature of the disease, as no outcome differences have been revealed between PC and HV type in patients with unicentric or multicentric disease (15).

The diagnosis of $\mathrm{CD}$ depends on pathological results. The clinical presentation of $\mathrm{CD}$ is characterized by asymptomatic or 
typical B-symptoms in conjunction with one or multiple tender or tender to touch lymph nodes. The aforementioned abnormal laboratory values may also be observed. These presentations may lead to the initial diagnosis of lymphoma $(10,23)$. Although elevated serum expression levels of IL-6, as well as circulating human herpes virus- 8 particles, may be useful for differential diagnosis, these two indicators are not widely nor commonly used in clinical practice (17). The size of the swollen lymph node in UCD, with a mean value of $5.7 \mathrm{~cm}$, may be another useful indicator, as the typical size of lymphoma lymph nodes is smaller (15).

Numerous pathophysiology studies on CD have demonstrated promising results that may guide treatment; however, current therapy remains largely based on published case reports only $(10,23,24)$. Talat et al $(15)$ reviewed 404 published cases and concluded that unicentric and multicentric diseases were separate entities, with a different response to treatment and long-term outcome. The biological behavior of UCD tends to be similar to benign disease, and the standard therapy for UCD is surgical excision, which has been proven to be curative if complete and en-bloc resection are performed (15). In our clinical practice, stromal tumors or lymphoma may be the initial working diagnosis, which may prompt a wedge resection of the tissue to achieve a classification. Under these circumstances, once a rapid pathological diagnosis of UCD has been established, complete resection of the lymph node and/or surrounding lymph nodes is performed to achieve a surgical cure. With the exception of lymphoma, accurate staging of the disease to eliminate the possibility of MCD should also be determined prior to surgery. By performing MDCT, it is possible to achieve an improved perioperative evaluation, which may aid selection of a laparotomy or a laparoscopic approach. In the present study, all the patients with UCD underwent complete surgical resection with free resection margins and survived with an excellent prognosis. A total of 5 patients who underwent laparoscopic surgery achieved faster recovery, which was due to the advantage of laparoscopy in gaining access to difficult areas of the abdomen, as well as improved visualization with bloodless dissection. No patients underwent complete excision received further therapies as no evidence of disease recurrence was discovered in these patients, which was consistent with other reports on the critical role of surgical excision in UCD treatment $(15,17)$.

The biological behavior of MCD has an invasive characteristic, with difficulties in controlling disease progression and a high rate of recurrence. Furthermore, MCD may result in secondary lesions, including plasma lymphoma or follicular dendritic sarcoma $(5,25)$. Therefore, MCD is a systemic disorder, with no definitive standard treatment regimens available. Although surgical removal may be necessary for patients with MCD in whom a pre-operative diagnosis is not possible, surgical intervention in the majority of cases provides no observable long-term benefit (15). Combined therapy is the most frequently used treatment, and certain regimens demonstrated promising results, whereas others did not (2). As the critical role of IL-6 in disease pathogenesis is being clarified, a molecular target of IL-6 may be a promising option. In a previous multinational, randomized, placebo-controlled study, intravenous administration of siltuximab at a dose of $11 \mathrm{mg} / \mathrm{kg}$ revealed promising results in patients with stable MCD (26). In the present study, 2 patients received molecular target therapy based on immunohistochemical results of positive cluster of differentiation (CD) 20 and IL-6. These 2 HIV-negative patients survived, which was consistent with the previous reports $(2,26)$. In patients with disease progression, more research is required and autologous hematopoietic stem cell transplantation may be a treatment option.

In conclusion, the present study investigated a number of patients with $\mathrm{CD}$ in a single institution. The characteristics of $\mathrm{CD}$ were investigated, in terms of symptoms, signs, laboratory examinations and pathological features, and we shared our experience in the treatment of CD. It was revealed that MDCT is beneficial for perioperative evaluation and that the laparoscopic approach is suitable for certain UCD patients with an abdominal tissue mass. In patients with MCD, molecular therapy targeting IL-6 and CD20, as well as autologous hematopoietic stem cell transplantation, may be useful.

\section{Acknowledgements}

The present study was funded by the National Natural Science Foundation of China (grant nos. 81500432, 81372364 and 81571563), the Fundamental Research Fund for the Central Universities (grant no. 021414380106), the China Postdoctoral Science Foundation (grant no. 2014M552695) and the People's Liberation Army Youth Culturing Project of Medical Sciences (grant no. 14QNP009).

\section{References}

1. Castleman B and Towne VW: Case records of the Massachusetts general hospital; weekly clinicopathological exercises; founded by Richard C. Cabot. N Engl J Med 251: 396-400, 1954.

2. El-Osta HE and Kurzrock R: Castleman's disease: From basic mechanisms to molecular therapeutics. Oncologist 16: 497-511, 2011.

3. Waterston A and Bower M: Fifty years of multicentric Castleman's disease. Acta Oncol 43: 698-704, 2004.

4. Keller AR, Hochholzer L and Castleman B: Hyaline-vascular and plasma-cell types of giant lymph node hyperplasia of the mediastinum and other locations. Cancer 29: 670-683, 1972.

5. Casper C: The aetiology and management of Castleman disease at 50 years: Translating pathophysiology to patient care. Br J Haematol 129: 3-17, 2005.

6. Yoshizaki K, Matsuda T, Nishimoto N, Kuritani T, Taeho L, Aozasa K, Nakahata T, Kawai H, Tagoh H, Komori T, et al: Pathogenic significance of interleukin-6 (IL-6/BSF-2) in Castleman's disease. Blood 74: 1360-1367, 1989.

7. Vinzio S, Ciarloni L, Schlienger JL, Rohr S, Méchine A and Goichot B: Isolated microcytic anemia disclosing a unicentric Castleman disease: The interleukin-6/hepcidin pathway? Eur J Intern Med 19: 367-369, 2008.

8. Reddy D and Mitsuyasu R: HIV-associated multicentric Castleman disease. Curr Opin Oncol 23: 475-481, 2011.

9. Shen XF, Guan WX, Cao K, Wang H and Du JF: Small bowel volvulus with jejunal diverticulum: Primary or secondary? World J Gastroenterol 21: 10480-10484, 2015.

10. Dispenzieri A: Castleman disease. Cancer Treat Res 142: 293-330, 2008.

11. Múzes G, Sipos F, Csomor J and Sréter L: Multicentric Castleman's disease: A challenging diagnosis. Pathol Oncol Res 19: 345-351, 2013.

12. Herrada J, Cabanillas F, Rice L, Manning J and Pugh W: The clinical behavior of localized and multicentric Castleman disease. Ann Intern Med 128: 657-662, 1998.

13. BonekampD,HortonKM,HrubanRHandFishmanEK:Castleman disease: The great mimic. Radiographics 31: 1793-1807, 2011.

14. Frizzera G: Castleman's disease and related disorders. Semin Diagn Pathol 5: 346-364, 1988. 
15. Talat N, Belgaumkar AP and Schulte KM: Surgery in Castleman's disease: A systematic review of 404 published cases. Ann Surg 255: 677-684, 2012.

16. Barker R, Kazmi F, Stebbing J, Ngan S, Chinn R, Nelson M, O'Doherty $\mathrm{M}$ and Bower M: FDG-PET/CT imaging in the management of HIV-associated multicentric Castleman's disease. Eur J Nucl Med Mol Imaging 36: 648-652, 2009.

17. Ye B, Gao SG, Li W, Yang LH, Zhao SH, Ma K, Zhu XL, Liu XY and Sun KL: A retrospective study of unicentric and multicentric Castleman's disease: A report of 52 patients. Med Oncol 27: $1171-1178,2010$.

18. Meador TL and McLarney JK: CT features of Castleman disease of the abdomen and pelvis. AJR Am J Roentgenol 175: 115-118, 2000.

19. Mangini M, Aiani L, Bertolotti E, Imperatori A, Rotolo N, Paddeu A, Uccella S, Carrafiello G and Fugazzola C: Parapancreatic Castleman disease: Contrast-enhanced sonography and CT features. J Clin Ultrasound 35: 207-211, 2007.

20. Noh OK, Lee SW, Lee JW, Kim SY, Kim CS, Choi EK, Kim JH and Ahn SD: Cases report of unicentric Castleman's disease: Revisit of radiotherapy role. Radiat Oncol J 31: 48-54, 2013.

21. Frizzera G, Banks PM, Massarelli G and Rosai J: A systemic lymphoproliferative disorder with morphologic features of Castleman's disease. Pathological findings in 15 patients. Am J Surg Pathol 7: 211-231, 1983.
22. Lee HJ, Jeon HJ, Park SG and Park CY: Castleman's disease of the spleen. World J Gastroenterol 21: 1675-1679, 2015.

23. Bowne WB, Lewis JJ, Filippa DA, Niesvizky R, Brooks AD, Burt ME and Brennan MF: The management of unicentric and multicentric Castleman's disease: A report of 16 cases and a review of the literature. Cancer 85: 706-717, 1999.

24. Soumerai JD, Sohani AR and Abramson JS: Diagnosis and management of Castleman disease. Cancer Control 21: 266-278, 2014.

25. Weisenburger DD, Nathwani BN, Winberg CD and Rappaport H: Multicentric angiofollicular lymph node hyperplasia: A clinicopathologic study of 16 cases. Hum Pathol 16: 162-172, 1985.

26. van Rhee F, Casper C, Voorhees PM, Fayad LE, van de Velde H, Vermeulen J, Qin X, Qi M, Tromp B and Kurzrock R: A phase 2, open-label, multicenter study of the long-term safety of siltuximab (an anti-interleukin-6 monoclonal antibody) in patients with multicentric Castleman disease. Oncotarget 6: 30408-30419, 2015. 